\title{
Context-Aware Ubiquitous Data Mining Based Agent Model for Intersection Safety*
}

\author{
Flora Dilys Salim ${ }^{1}$, Shonali Krishnaswamy ${ }^{1}$, Seng Wai Loke ${ }^{1}$, \\ and Andry Rakotonirainy ${ }^{2}$ \\ ${ }^{1}$ Caulfield School of Information Technology, Monash University, \\ 900 Dandenong Road, Caulfield East, VIC 3145, Australia \\ \{Flora.Salim, Shonali.Krishnaswamy, \\ Seng.Loke\} @infotech.monash.edu.au \\ ${ }^{2}$ Centre for Accident Research and Road Safety Queensland, \\ Queensland University of Technology, Beams Road, Carseldine, QLD 4034, Australia \\ $r$. andryaqut. edu. au
}

\begin{abstract}
In USA, 2002, approximately 3.2 million intersection-related crashes occurred, corresponding to 50 percent of all reported crashes. In Japan, more than 58 percent of all traffic crashes occur at intersections. With the advances in Intelligent Transportation Systems, such as off-the-shelf and in-vehicle sensor technology, wireless communication and ubiquitous computing research, safety of intersection environments can be improved. This research aims to investigate an integration of intelligent software agents and ubiquitous data stream mining, for a novel context-aware framework that is able to: (1) monitor an intersection to learn for patterns of collisions and factors leading to a collision; (2) learn to recognize potential hazards in intersections from information communicated by road infrastructures, approaching and passing vehicles, and external entities; (3) warn particular threatened vehicles that are approaching the intersection by communicating directly to the in-vehicle system.
\end{abstract}

\section{Background}

In spite of the advancement of state-of-the-art technologies being implemented in vehicles and on the road over the years, the annual toll of human loss caused by intersection crashes has not significantly changed in more than 25 years, regardless of improved intersection design and more sophisticated ITS technology [21]. Intersections are among the most dangerous locations on U.S. roads [7]. In 2002, USA, approximately 3.2 million intersection-related crashes occurred, corresponding to 50 percent of all reported crashes. 9,612 fatalities (22 percent of total fatalities) [21] and roughly 1.5 million injuries and 3 million crashes took place at or within an intersection [22]. Yearly, 27 percent of the crashes in the United States take place at intersections [7]. In Japan, more than 58 percent of all traffic crashes occur at intersections. Intersections-

* The work reported in this paper has been funded in part by the Co-operative Research Centre Programme through the Australian Government's Department of Education, Science and Training. 
related fatalities in Japan are about 30 percent of all Japanese traffic accidents, and those fatal crashes mainly happen at intersections without traffic signals [7].

The complexity of intersections is due to various characteristics of intersections [1, 7, 19], which are as follows: different intersection shapes, number of intersection legs, signalized/ unsignalized, traffic volume, rural / urban setting, types of vehicles using the intersection, various average traffic speed, median width, road turn types, and number of lanes. From those characteristics that pertain to intersection collisions, a driving assistance system for intersection is highly needed, particularly one that is able to warn driver for potential threats or collisions. Given the uniqueness of each intersection, an intelligent system for intersection safety should be able to adapt to different characteristics of an intersection [19].

The advances in sensor technology and the need for intelligence, dynamicity, and adaptability in ITS have motivated the research of Context-Awareness, Multiagent Systems, and Data Mining for Intelligent Transportation Systems as discussed in Section 2. Section 3 discusses the model we propose to address the issues of intersection safety. Section 4 concludes the paper and outlines future work of the project.

\section{Related Work}

Subsection 2.1 reviews existing research projects in intelligent software systems, such as Context-Awareness, Multiagent Systems, and Data Mining, which have been utilized to advance Intelligent Transportation Systems. Subsection 2.2 discusses the existing approaches in intersection collision warning and/or avoidance systems.

\subsection{Intelligent Software Systems}

Context-aware applications observe the "who's, where's, when's, and what's" of entities and use this information to find out "why" a situation is happening [2]. With the availability of context information, an application can then use it to adapt to environment changes. The research areas of context-awareness in ITS include smart autonomous cars [17, 18] and traffic monitoring [11].

An agent is autonomous intelligent program acting on behalf of the user [24]. A multiagent system (MAS) is a collection of agents that communicate with each other and work together to achieve common goals with a certain measure of reactivity and/or reasoning [24]. There have been considerable ITS projects using the notion of agents, such as for controlling and managing traffic in intersections $[3,6,10]$.

Given that there are considerable amount of data from the in-vehicles and roadside sensors, clearly, it is essential to make sense of the sensors data. Data mining is the development of methods and techniques to gain knowledge from data by pattern discovery and extraction [4]. Data analysis techniques are necessary for processing information both on roadside and in vehicle situations [16]. However, data mining and machine learning techniques require high computational resources as knowledge is discovered from the analysis of huge data storages. Learning from data streams in ubiquitous environment is enabled by Ubiquitous Data Mining (UDM), which is the analysis of data streams to discover useful knowledge on mobile, embedded, and ubiquitous devices [9]. UDM have been used to monitor vehicle's health and driver's characteristics in moving vehicles [13] and to identify drunk-driving behavior [12]. 
The above mentioned technology in ubiquitous computing enables more sophisticated ITS applications. However, after reviewing those research projects, none has addressed a holistic approach for intersection safety.

\subsection{Intersection Collision Warning and/or Avoidance Systems}

Intersection collision warning and avoidance systems are categorized as either vehicle-based, infrastructure-only or as infrastructure vehicle cooperative [5, 22]. Vehicle-based systems rely only on in-vehicle sensors, processors, and interface to detect threats and produce warnings [22]. Infrastructure-only systems rely only on roadside warning devices to inform drivers [5]. Cooperative systems communicate information straight to vehicles and drivers. The main advantage of cooperative systems rests in their potential to improve the interface to the driver, and thus to almost guarantee that a warning is received.

Existing Intersection Collision Warning Systems as those described in [5, 8, 19, 20, 23 are still infrastructure-only system, and are limited in certain aspects:

1. Warning messages are less effective as they are only displayed on the roadside.

2. There is no communication means that exists between road infrastructure and vehicles, and therefore, no exchange of useful information between them.

3. Information about intersection might not be comprehensive as the only data source is roadside sensors.

4. The systems are mostly reactive. Although reactive trait is required; however, deliberative reasoning aspect can supplement and enhance these systems.

5. Each system is built for a particular intersection or an intersection type, and therefore each application requires a field study on that intersection.

Vehicle-based intersection collision warning systems [15] are fairly effective for a single vehicle. However, in an intersection, a cooperative system is a preferred solution as it is very important to communicate foreseen threats to other vehicles.

Research initiatives in developing cooperative system for intersection safety such as $[14,22]$ have recently commenced. However, these projects do not mention the techniques to discover crash patterns and pre-crash behavior associations, which are essential to detecting and reacting to potential threats. A generic framework that is able to automatically adapt to various types of intersections is also required for efficiency of deployment; however, these projects have not addressed this issue.

There is a project that uses multiagent system for intersection collision warning system [22]; however, it only implements vehicle-to-vehicle cooperation for intersection safety. Threat detection relies on information (location, velocity, acceleration) shared by other vehicles. Useful information from external sources such as the infrastructure and environment are not incorporated. Another limitation is that the agent architecture is reactive; there is no learning to gain new knowledge that can improve the system.

Therefore we suggest an integration of multi-agent systems and ubiquitous data mining notions to a hybrid intersection safety model that can be applied to any intersection. The elucidation and model of our approach is described in the Section 3. 


\section{Proposed Model}

Subsection 3.1 outlines the requirements of the model for intersection safety management. Subsection 3.2 explains our model to answer those requirements.

\subsection{Model Requirements}

There is a need for a cooperative intersection collision warning and avoidance system that addresses the following challenges:

1. An intersection safety model that is able to detect high risk situations and foresee threats in particular intersections is required. Given that there is considerable amount of sensor data in cars and infrastructures, there is an opportunity to reason and use this data to develop patterns and associations that can help in better understanding of high risk situations and behaviors that lead to crashes. While current systems tend to be reactive to situations, there is increased recognition $[3,14,22]$ that reasoning and learning can be integrated to supplement reactivity.

2. As each intersection is unique, the profile of high risk situations in one intersection is different from another, therefore, a generic model that is able to adapt to particular intersections over a period of time is required. Each system in different intersections should have a knowledge that is applicable only within its locality. This knowledge is gained through reasoning and learning. Hence, this approach alleviates the inefficiency of the current method of developing different intersection collision warning and avoidance systems for different intersections [1, 7, 19].

3. There is a necessity for exchange of information and knowledge between intersection infrastructure and vehicles and also for vehicle-to-vehicle communication. This is due to the need for a comprehensive understanding of a particular intersection so that the system is able to act or respond better to a hazardous situation.

This research aims to investigate an integration of intelligent software agents, ubiquitous data stream mining, for a novel context-aware framework that is able to:

1. monitor an intersection to learn for patterns of collisions and factors leading to a collision using ubiquitous data stream mining;

2. learn to recognize potential hazards in intersections from information communicated by road infrastructures, approaching and passing vehicles, and external entities using a layered agent architecture;

3. warn particular threatened vehicles that are in the intersection by communicating directly to the in-vehicle system with multi-agent communication principles.

The goal is feasible due to the recent advances in ITS sensor technology that allows real-time data from in-vehicle and traffic sensors to become more accessible.

\subsection{Model Description}

This research brings together Multi-Agent Systems with Ubiquitous Data Mining to develop a context-aware model that addresses for cooperative intersection collision warning and avoidance systems.

Multiagent technology is very fitting for coordination of entities in intersections. The abstraction of independent, autonomous entities that are able to communicate with 
other entities and make independent decisions maps eminently to the situation of an on-road scenario. Each entity can be represented by an intelligent agent. Communication among those entities is made possible through agent communication language. Accordingly, we need to decide on which agent architecture is the most appropriate to answer the challenges in the Section 1. According to [24], there are four classifications of agents based on their architectures: logic, reactive, BDI, and layered agents. As agent's layered architecture is designed for balance of mutual effectiveness of reactivity and reasoning, thus we view it as appropriate to adopt this architecture for the basis of the model of agents for intersection safety system. Such model allows retaining the element of reactivity while incorporating the potential to reason and learning.

The question now remains as to how the reasoning and learning is accomplished. We view Ubiquitous Data Mining (UDM) as suitable in this context. A system that is deployed to continuously monitor an intersection must necessarily be able to operate in a ubiquitous resource-constrained environment. The information delivered to the systems will be from a myriad of sensors that continuously and rapidly stream data to the systems. Given this content, it is evident that UDM is a suitable option and one that can facilitate incremental learning. The question remains that while the general principals of UDM are appropriate for our research, the specifics and modalities of the learning process and the algorithms suited to this application need to be investigated and developed as part of this research.

Therefore, the model we propose is: A context-aware multi-agent framework with an integration of layered agent architecture and ubiquitous data mining for intersection safety. The subsection 3.2.1 discusses the internal model of agents, while the subsection 3.2.2 discusses the interaction model of our multiagent system.

\subsubsection{Agent Model}

For each agent in the framework, we propose a novel hybrid agent model: Ubiquitous Data Mining based Layered Agent (UDMLA), as displayed in Figure 1.

The theoretical model consists of three layers, which are described as follows:

1. Reactive layer as the bottom layer. It has sensors, communication components, and actuators that accept sensory data input and generate responses. It performs information exchanges with other agents or external parties and performs the task of issuing notifications. Reactive layer possesses knowledge based rules for generating actions or responses. The characteristics of the knowledge in this layer are stable (unchanging for an extended period of time) and highly reliable or have high levels of confidence.

2. Training layer is intended to test new knowledge from the higher layer. Data received from reactive layer are passed to the higher layer for reasoning. This layer is designed to train untested knowledge that is passed from reasoning layer by data mining techniques for training datasets, solve conflict in untested knowledge by confidence measurement, recognize failures and learn from it by passing the information back to the reasoning layer. This layer possesses knowledge with moderate confidence as the knowledge still needs to be tested. When this knowledge has reached acceptable levels of stability or confidence, it is passed to the reactive layer for initiating actions based on events that conform to these patterns.

3. Reasoning layer contains UDM algorithm that extracts information from streams of data to recognize new knowledge such as in form of patterns and associations. 


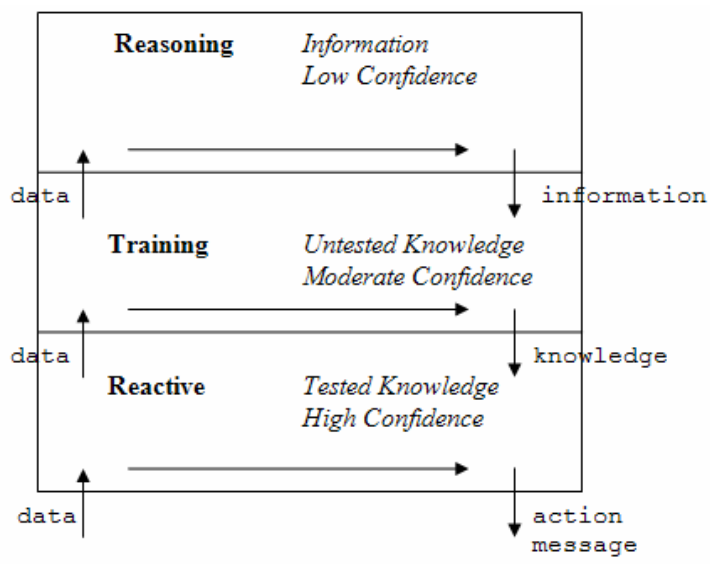

Fig. 1. UDM based Layered Agent (UDMLA) model

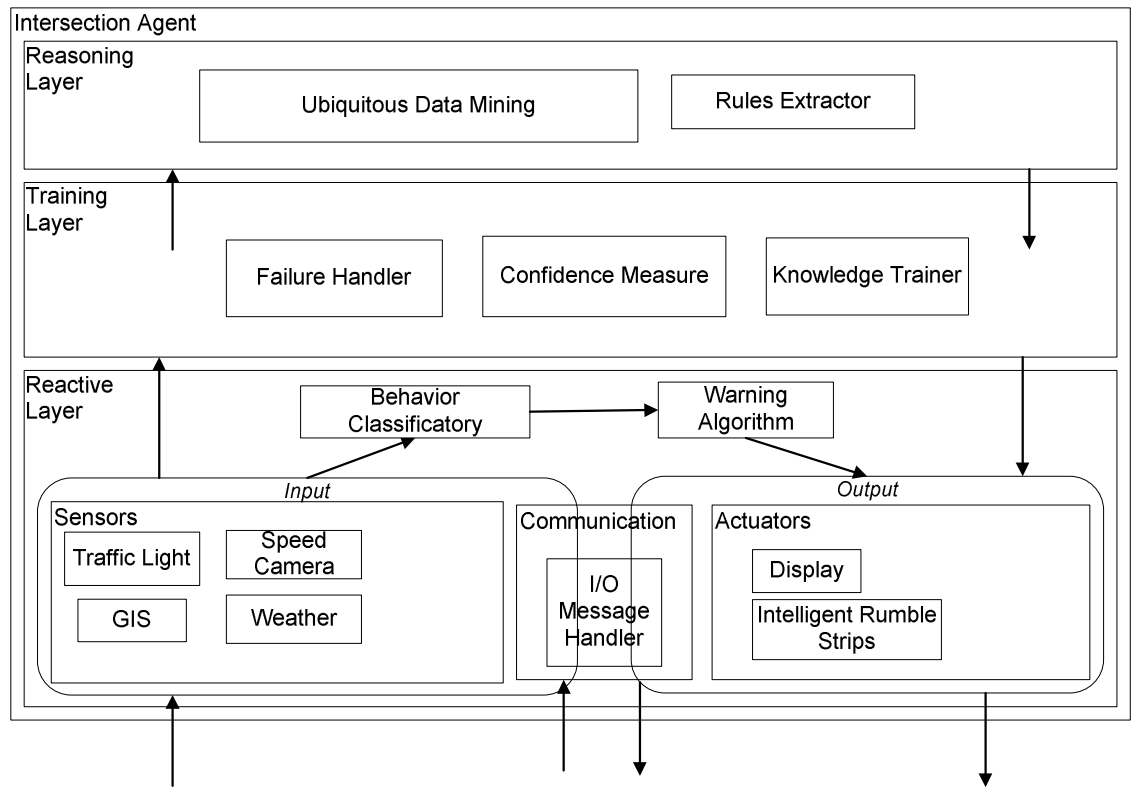

Fig. 2. The internal architecture of an intersection agent

Each layer has a confidence measure to check whether data entering the layer can be treated within certain levels of confidence for specific purposes such as for generating actions or training; otherwise, data will be passed into higher layers for reasoning. This approach facilitates knowledge evolution within the layers of the agent; hence, the agent is improving its intelligence over a period of time.

To our knowledge, a model of intelligent agent architecture that accommodates Ubiquitous Data Mining is novel. The UDMLA model is applicable to other applica- 
tion domains that require reactivity along with deliberation to cope with a fast changing environment.

Figure 2 shows an application of UDMLA model for a single intersection agent. Input to the reactive layer of an intersection agent can come from sensory inputs and also from different sources, such as from vehicle agents and external parties such as traffic bureau. The input data is checked against behavior classificatory to be validated whether it falls into one of the dangerous behavior categories. If it does, the data will be passed on to warning algorithm that will take an action depends on the rate of danger a situation carries.

Every input to the reactive layer is also passed into the next upper layer, which is the training layer. The training layer assesses the input and remeasures the confidence of knowledge by calculating and comparing the number of valid and invalid matched data items. For example, if knowledge to be tested states that a car that a travel approaching the vicinity is making a direct left turn without first yielding right-of-way, and another car from the opposite side of intersection, with the distance less than 25 meters, is approaching with the average speed of $50 \mathrm{~km} / \mathrm{h}$, crash will happen. In this case, the crash will likely occur in 1.8 second (3600 seconds $\div(50000 \mathrm{~m} \div 25 \mathrm{~m})$ ) Say that this knowledge has 3 valid occurrences out of 4 total occurrences $(75 \%$ confidence). A new data item that falls within the same situation adds the confidence of the knowledge to be 4 out of 5 ( $80 \%$ confidence). A confidence threshold is given to this layer, that before a knowledge can be passed into the reactive layer it must reach a certain level of confidence, for example $90 \%$ confidence. If there is a failure in warning relevant vehicles (i.e. crash happens), failure handler will store the case and test next relevant data items whether the correct rule is the negation of the current rule or is a fuzzy rule derived from both the current rule and its negation.

The top layer is the reasoning layer where all input data are being learned and studied by Ubiquitous Data Mining techniques to find patterns of intersection crashes and dangerous situations and driver behaviors that lead to each crash category. Rules for classifying situations are also being extracted here after clusters of crash patterns are found, so that dangerous situations can be detected instantly. Every new rule extracted is transferred to the training layer to be tested with new data items over a period of time.

One example of a scenario that is examined by an intersection agent is a situation of a small size car that is approaching the intersection with the speed of $40-60 \mathrm{~km} / \mathrm{h}$ without decelerating to beat the yellow traffic light before it turns to red in 0.5 second. A near side-collision event occurs as a car from the other side of intersection suddenly puts on the brakes within the distance of 0.5 meter from the car that violates the red light signal. This event is then recorded with all the attributes to be clustered and classified by UDM algorithm. The clustering of UDM uses initial clusters depends on intersection types and crash patterns described by previous studies. For example, for a cross intersection [15], the initial clusters are: (1) across path turn; (2) perpendicular paths with no violation of traffic control; (3) perpendicular paths with violation of traffic control; (4) premature intersection entry scenario. Driving behaviors and attributes in each of the cluster will then be mapped against five stages of driving, which are "normal, warning, collision avoiding, collision imminent, and collision past" [24]. The warning algorithm treats each stage of driving differently by issuing different level of warning. The number of crash patterns will change according to the usage behaviors and characteristics of the intersection. Hence, the intersection agent is context-aware, and able to adapt to different kinds of intersections due to its learning capabilities. 
Vehicle agents are using reactive agent architecture as immediate actions should be taken in response to warning messages from the intersection agent and possibly from other vehicle agents. Vehicle agents only carry knowledge that are tested and has a high level of confidence. This knowledge is communicated by the intersection agent. The multiagent interaction model used for the system is discussed in the next subsection 3.2.2.

\subsubsection{Multiagent Interaction Model}

The multi-agent system consists of a stationary agent in an intersection and also mobile agents in vehicles and is capable of discovering knowledge from streams of data from various sources such as sensors, traffic bureau and weather bureau. Multi-agent system will be applied on the whole intersection-vehicle system. Each vehicle will have at least one vehicle agent, and every intersection will have at least one stationary agent. These agents will then communicate and work together to achieve their common goals using their individual and shared knowledge delivered from ubiquitous data mining. As a result, the system will be more knowledgeable over periods of time. If a vehicle or a driver has unacceptable behaviors that will risk the other road users, mobile agents will warn the stationary agent in an adjacent intersection. If a danger for collision is foreseen by either the stationary agent at the intersection, warnings will be sent to all relevant vehicles. An agent that resides in each vehicle will then act accordingly to the warning message and also to the situation of the vehicle and driver. This architecture is general for all kinds of intersections, as each intersection will have its own set of localized knowledge. This is due to the different crash patterns that exist because of the situation difference, such as intersection shape, location, volume usage, and presence of different traffic signals. As a result, this infrastructure safety architecture is also a context-aware system that knows about its current situation and knows how to react and adapt to different situations. The intersection agent operates within its zone of influence.

A zone of influence is the spatial domain that determines the region of authority of an intersection agent to coordinate vehicle agents in the approaching and passing vehicles. Knowledge about an intersection that is possessed by an intersection agent is specific within the boundaries of the zone of influence. Once a vehicle enters a zone of influence, it broadcasts its sensor data to the intersection agent that resides in the zone of influence. The intersection agent will then transfer its knowledge about the intersection to the vehicle for the knowledge base of the vehicle agent's warning algorithm. Warnings are produced mainly from the vehicle agent when the agent detects the driver is executing dangerous driving maneuvers. However, warnings are also produced from the intersection agent and sent to relevant vehicles that are going to be affected, as at some situations where multiple cars are involved, it is only the intersection agent that is able to detect and analyze the situation well. In the intersection agent, the zone of influence is managed by I/O message handler in the reactive layer.

Our architecture for intersection collision warning and avoidance system enables vehicle-to-vehicle communication and vehicle-to-infrastructure communication via agent communication protocol. The necessity of applying data processing and analysis techniques to assess different situations in an intersection is satisfied by having ubiquitous data mining that is learning from sensors information. Another benefit of this approach is that it is a scalable solution as there is an automatic localization to specific intersections. 


\section{Conclusion and Future Work}

We have proposed Ubiquitous Data Mining based Layered (UDMLA) model for cooperative intersection-vehicle safety: an integration of layered agent architecture with ubiquitous data mining and context-awareness for intersection safety with the notion of support and confidence of data mining for knowledge evolution of an agent.

Our contribution to research in road safety is a generic intersection safety model that can adapt to specific intersections. We are currently implementing the UDMLA model on a computer based simulation.

\section{References}

1. Arndt, O. K.: Relationship Between Unsignalised Intersection Geometry and Accident Rates, School of Civil Engineering, Queensland University of Technology, PhD Thesis (2003)

2. Dey, A. K. and Abowd, G. D.: Towards a Better Understanding of Context and ContextAwareness, 1st International Symposium on Handheld and Ubiquitous Computing, GVU Technical Report GIT-GVU-99-22 (1999)

3. Dresner, K. and Stone, P.: Multiagent Traffic Management: An Improved Intersection Control Mechanism, the Proceedings of The Fourth International Joint Conference on Autonomous Agents and Multi Agent Systems (AAMAS '05), Utrecht, Netherlands (2005)

4. Fayyad, U., Piatetsky-Shapiro, G., Smyth, P.: From Data Mining to Knowledge Discovery in Databases, AI Magazine Vol. 17, No. 3 (1996)

5. Ferlis, R. A.: Infrastructure Intersection Collision Avoidance, Intersection Safety Conference, Milwaukee, WI (2001)

6. France, J. and Ghorbani, A. A.: A Multi-Agent System for Optimizing Urban Traffic, Proc. of IEEE/WIC International Conference on Intelligent Agent Technology (IAT 2003), Halifax, Nova Scotia, Canada (2003)

7. Frye, C.: "International Cooperation to Prevent Collisions at Intersections", Public Roads Magazine, Vol. 65, No. 1, July-August 2001, Federal Highway Administration, USA (2001)

8. Funderburg, K. A.: "Update on Intelligent Vehicles and Intersections", Public Roads Magazine, Vol. 67, No. 4, January-February 2001, Federal Highway Administration, USA (2004)

9. Gaber, M. M., Krishnaswamy, S., and Zaslavsky, A.: Ubiquitous Data Stream Mining, Current Research and Future Directions Workshop, in conjunction with The Eighth PacificAsia Conference on Knowledge Discovery and Data Mining, Sydney, Australia (2004)

10. Gabric, T., Howden, N., Norling, E., Tidhar, G., and Sonenberg, E.: Agent-oriented design of a traffic flow control system, University of Melbourne Department of Computer Science Technical Report 94/24 (1994)

11. Harrington, A., and Cahill, V.: Route Profiling - Putting Context To Work, Proceedings of the 19th ACM Symposium on Applied Computing (SAC 2004), Nicosia, Cyprus (2004) $1567-1573$

12. Horovitz, O., Gaber, M, M., and Krishnaswamy, S.: Making Sense of Ubiquitous Data Streams: A Fuzzy Logic Approach, to appear in the Proceedings of the 9th International Conference on Knowledge-based Intelligent Information \& Engineering Systems 2005 (KES 2005), Melbourne, Australia (2005)

13. Kargupta, H., Bhargava, R., Liu, K., Powers, M., Blair, P., Bushra, S., Dull, J., Sarkar, K., Klein, M., Vasa, M. and Handy, D.: VEDAS: A Mobile and Distributed Data Stream Mining System for Real-Time Vehicle Monitoring, Proceedings of the SIAM International Data Mining Conference, Orlando (2004) 
14. Lages, U.: INTERSAFE - New European Approach for Intersection Safety, funded by the European Commission in 6th Framework Program, 11th World Congress on ITS, Nagoya, Japan (2004)

15. Lloyd, M., Pierowicz, J., Jocoy, E., Pirson, B., Bittner, A.: Intersection Collision Avoidance Using Its Countermeasures. Task 9: Final Report: Intersection Collision Avoidance System Performance Guidelines, U. S. Department of Transportation, National Highway Traffic Safety Administration (2000)

16. Miller, R., Huang, Q.: An Adaptive Peer-to-Peer Collision Warning System, Vehicular Technology Conference (VTC), Birmingham, Alabama (2002)

17. Oliver, N. and Pentland, A.: Graphical Models for Driver Behavior Recognition in a SmartCar, Proceedings of IEEE International Conference on Intelligent Vehicles, Detroit, Michigan (2000)

18. Sivaharan, T., Blair, G., Friday, A., Wu, M., Duran-Limon, H., Okanda, P., and Sørensen, C-F.: Cooperating Sentient Vehicles for Next Generation Automobiles, ACM MobiSys International Workshop on Applications of Mobile Embedded Systems, Boston (2004)

19. Stubbs, K., Arumugam, H., Masoud, O., McMillen, Veeraraghavan, H., Janardan, R., Papanikolopoulos, N.: A Real-Time Collision Warning System for Intersections, Proceedings of Intelligent Transportation Systems America, Minneapolis (2003)

20. U.S. Department of Transportation - Federal Highway Administration: Intersection Collision Warning System, April 1999, http://www.tfhrc.gov/safety/pubs/99103.pdf (1999)

21. U.S. Department of Transportation - Federal Highway Administration, Institute of Transportatio Engineers: Intersection Safety Briefing Sheet, April 2004, http://www.ite.org/library/IntersectionSafety/BreifingSheets.pdf (2004)

22. U.S. Department of Transportation: Cooperative Intersection Collision Avoidance System, http://www.its.dot.gov/initiatives/initiative2.htm (2005)

23. Veeraraghavan, H., Masoud, O., and Papanikolopoulos, N.: Vision-based Monitoring of Intersections, Proceedings of IEEE Intelligent Transportation Systems Conference (2002)

24. Wooldridge, M.: "Intelligent Agents", Multiagent systems: A modern approach to distributed artificial intelligence, Chapter 1, Weiss, G. (Ed.), The MIT Press (1999) 27 -77 